\title{
Suplementação com gordura protegida para vacas de corte desmamadas precocemente mantidas em pastagem natural
}

\author{
[Protected fat supplementation for early weaned beef cows maintained in nature pasture] \\ M.F. Silveira ${ }^{1}$, J. Restle ${ }^{3}$, D.C. Alves Filho ${ }^{1}$, R.L. Missio ${ }^{2}$, P.A.M.M. Donicht ${ }^{1}$, L.R. Segabinazzi ${ }^{1}$, \\ A.M. Callegaro ${ }^{1}$, G. Joner ${ }^{1}$ \\ ${ }^{1}$ Universidade Federal de Santa Maria - UFSM - Santa Maria, RS \\ ${ }^{2}$ Universidade Estadual do Estado de São Paulo - Unesp - Campus Jaboticabal, SP \\ ${ }^{3}$ Universidade Federal de Goiás - UFG - Goiânia, GO
}

\begin{abstract}
RESUMO
Avaliaram-se os desempenhos produtivo e reprodutivo de vacas de corte, bem como o desempenho de seus bezerros, de acordo com os tratamentos alimentares: PRE: suplementação com gordura protegida (GP) 45 dias antes do parto; PREPOS: suplementação com GP 45 dias antes do parto e 63 dias pós-parto; POS: suplementação com GP 63 dias pós-parto; PN: sem suplementação. O desempenho produtivo das vacas não foi influenciado pelo manejo alimentar $(\mathrm{P}>0,05)$, exceto para o escore da condição corporal (ECC) no final do período de acasalamento, que foi mais baixo para as vacas do PRE e do PREPOS, sendo que esta última apresentou ECC semelhante ao das vacas do POS e do PN. O intervalo entre partos foi menor para as vacas do tratamento PREPOS - 376 dias -, não diferindo das vacas do PN - 383 dias. As vacas do PREPOS desmamaram $4,4 \%$ mais quilos de bezerro para cada $100 \mathrm{~kg}$ de vaca ao parto $22,6 \mathrm{~kg}$ - do que as vacas do PRE e do POS - 21,6kg e 21,6kg, respectivamente - e 8,4\% mais quilos de bezerro para cada $100 \mathrm{~kg}$ de vaca ao parto do que as vacas mantidas em pastagem nativa $-20,7 \mathrm{~kg}$. A suplementação com gordura protegida durante os períodos pré e/ou pós-parto não afeta o desempenho de vacas e bezerros.
\end{abstract}

Palavras-chave: bovino de corte, eficiência reprodutiva, peso ao nascer, peso ao parto, produção de leite

\begin{abstract}
The objective of this work was to evaluate the productive and reproductive performance of beef cows, as well as the performance of their calves according to the following dietary treatments: PRE: supplemented with protected fat $(P F)$ during 45 days prepartum; PREPOS: supplemented with PF during 45 days prepartum and 63 days postpartum; POS: supplemented with PF during 63 days postpartum; PN: without supplementation. The productive performance of cows was not influenced by feed management $(P>0.05)$, except for body condition score (BCS), which was lower for PRE and PREPOS cows at the end of mating season, with the latter cows having similar BCS POS and PN. The calving interval (CI) was shorter for cows supplemented in PREPOS - 376 days -, and did not differ in cows maintained in PN 383 days. Supplemented PREPOS cows weaned $4.4 \%$ more pounds of calf per 100kg of cow at calving $22.6 \mathrm{~kg}$ - than the PRE and POS cows - 21.6kg and 21.6kg, respectively - and 8,4\% more pounds of calf per 100 of cow at calving than the cows maintained in native pasture $-20.7 \mathrm{~kg}$. The fat protected supplementation during pre and/or postpartum periods did not affect the performance of cows and calves.
\end{abstract}

Keywords: beef cattle, reproductive efficiency, birth weight, parturition weigth, milk production

Recebido em 2 de março de 2012

Aceito em 23 de agosto de 2013

E-mail: mg_flor@yahoo.com.br 


\section{INTRODUÇÃO}

Na região Sul do Brasil, as matrizes alimentamse basicamente de pastagens nativas, as quais estão sujeitas às oscilações no valor nutritivo e na produção de forragem, que afetam consequentemente seu desempenho reprodutivo. Para promover maior aporte nutricional às matrizes, a suplementação lipídica pode ser uma alternativa, já que a porcentagem de gordura na dieta de ruminantes mantidos somente em pasto está entre 1 e $4 \%$ na matéria seca (MS). De acordo com Palmquist e Jenkis (1980), a gordura deve ser limitada de 5 a $6 \%$ na MS da dieta, pois valores mais altos podem afetar negativamente a digestibilidade da fibra e o consumo de alimentos. Uma forma de fornecer suplementação lipídica sem prejuízo aos animais seria a utilização de gordura protegida. $\mathrm{O}$ uso de sais de cálcio de ácidos graxos na dieta aumenta a densidade energética da dieta, refletindo em incremento na produção de leite (Nörnberg et al., 2006) e com efeitos positivos diretos na reprodução (Lopes et al., 2009).

O período para fornecer a suplementação lipídica é, ainda, motivo de muita discussão, pois os resultados que avaliam o pré-parto ou o pósparto são diversos, tanto positivos como negativos para ambos os períodos (Funston, 2004). Vacas de corte sob restrição alimentar durante o período pré-parto apresentam perda de peso e diminuição na condição corporal ao parto, resultando em um longo intervalo parto-primeiro cio (Colazo et al., 2009). Já Richrads et al., (1986) encontraram longo intervalo partoprimeiro cio quando as vacas sofreram subnutrição no pós-parto.O equilíbrio de energia durante as primeiras semanas de lactação é altamente correlacionado com o intervalo partoprimeiro cio (Butler, 2000). Além disso, esses períodos influenciam a capacidade produtiva de leite das vacas, que é um dos principais fatores que afetam o peso ao desmame dos bezerros (Cérdotes et al., 2004).

Durante a fase de cria, a eficiência produtiva do rebanho bovino de corte é definida como a conversão de energia alimentar em peso de bezerro desmamado (Jenkis e Ferrel, 1992). No entanto, poucos resultados são verificados sobre a avaliação biológica do sistema de cria ao se estudar o manejo nutricional, sendo que a maioria dos estudos avaliou o grupo racial das vacas (Ribeiro et al., 2001; Restle et al., 2004a).

O objetivo deste trabalho foi avaliar o fornecimento de gordura protegida durante os períodos pré e/ou pós-parto nos desempenhos reprodutivo, produtivo e na produção e composição do leite de vacas de corte mantidas em pastagem natural, bem como o desempenho produtivo de seus bezerros.

\section{MATERIAL E MÉTODOS}

O estudo foi realizado no Laboratório de Bovinocultura de Corte, pertencente ao Departamento de Zootecnia da UFSM, entre agosto de 2008 e maio de 2009 . Foram utilizados 86 pares de vacas e bezerros, mestiços NeloreCharolês, sendo que as vacas apresentavam, em média, cinco anos de idade.

O suplemento utilizado foi sais de cálcio de ácidos graxos (Megalac ${ }^{\circledR}$-E, Química Geral do Nordeste), na quantidade diária de $60 \mathrm{~g} /$ vaca no período pré-parto e $100 \mathrm{~g} /$ vaca no período pósparto, fornecido juntamente com $80 \mathrm{~g} / \mathrm{vaca} / \mathrm{dia}$ de sal mineral com $8 \%$ de fósforo. A recomendação, segundo o fabricante da gordura protegida, é utilizar 50-120g/cabeça/dia três semanas antes do parto e, após o parto, aumentar a dose para $150 \mathrm{~g} / \mathrm{cabeça} / \mathrm{dia}$ até atingir 250$900 \mathrm{~g} / \mathrm{cabeça/dia.} \mathrm{Esta} \mathrm{recomendação} \mathrm{é} \mathrm{para}$ vacas de raças leiteiras de alta produção, contudo as vacas utilizadas no presente estudo eram vacas de raças de corte. Dessa forma, utilizou-se a menor recomendação sugerida pela empresa. As vacas foram suplementadas durante 45 dias antes do parto (PRE), 45 dias antes e 63 dias pós-parto (PREPOS), 63 dias pós-parto (POS), ou não receberam a suplementação $(\mathrm{PN})$, caracterizando os tratamentos alimentares testados.

A área experimental utilizada correspondeu a 88 hectares, divididos em três potreiros de 29,3ha. A cada 14 dias foi realizado rodízio dos animais para minimizar o efeito do potreiro. A lotação média foi de $0,6 \mathrm{UA} / \mathrm{ha}$. A massa de forragem (MF) disponível foi determinada a cada 28 dias pela técnica da estimativa visual em dupla amostragem (Wilm, 1944). Para a determinação da taxa de acúmulo de forragem (TAD, kg/ha/dia MS), foram usadas cinco gaiolas de exclusão ao pastejo por potreiro. A forragem obtida dos cortes foi homogeneizada. Posteriormente, 
retirou-se amostra, que foi seca em estufa de $55^{\circ} \mathrm{C}$ durante pelo menos 72 horas, moída em peneira com porosidade de $1 \mathrm{~mm}$ e armazenada para posteriores análises bromatológicas.

O período de parição foi de setembro a novembro. $\mathrm{O}$ peso ao parto da vaca e o peso ao nascimento do bezerro foram obtidos nas primeiras 24 horas após o parto, e após, com intervalos de 21 dias até os 63 dias pós-parto momento do desmame -, com variação de \pm 3 dias, de acordo com a data do parto. Adicionalmente à pesagem, foi avaliado o escore de condição corporal das vacas, seguindo uma escala de 1 a 5: 1=muito magra, 2=magra, 3 =média, 4=gorda e 5=muito gorda (Lowman et al., 1973).

As medidas de eficiência produtiva e reprodutiva foram calculadas da seguinte forma: quantidade de quilos de bezerros desmamados para 100 quilos de vacas paridas: EFPARTO = $(\mathrm{P} 63 / \mathrm{PVP})^{*} 100$, em que $\mathrm{P} 63=$ peso vivo $(\mathrm{kg})$ dos bezerros ao desmame; $\mathrm{PVP}=$ peso vivo $(\mathrm{kg}) \mathrm{da}$ vaca ao parto; quantidade de quilos de bezerros desmamados para 100 quilos de vacas ao desmame (PVD): $\quad$ EFDESMAME= (P63/PVD)*100; quantidade de quilos de bezerros desmamados por unidade de tamanho metabólico ao parto das vacas ajustado para as taxas de prenhez: EFRTM $=\left(\mathrm{P} 63 /(\mathrm{PVP})^{0,75}\right) * \operatorname{taxa}$ de prenhez, em que $\mathrm{PVP}^{0,75}=$ peso vivo $(\mathrm{kg}) \mathrm{da}$ vaca por unidade de tamanho metabólico. Após o desmame, as vacas foram separadas por grupo genético e expostas aos touros até o final do período de acasalamento, totalizando 90 dias, momento em que foi obtido o peso final de monta das vacas e seu ECC. O diagnóstico de prenhez, mediante a palpação retal, foi realizado, em média, após 60 dias do final do período de acasalamento. $\mathrm{O}$ intervalo entre partos (IP) foi calculado por meio da diferença da data do parto em 2008 e da data do parto subsequente, em 2009.

A coleta de leite foi realizada aos 21, 42 e 63 dias após o parto, com variação de \pm 3 dias. $\mathrm{O}$ desmame dos bezerros foi aos 63 dias de idade. Para a coleta de leite, foram utilizadas 11 vacas por tratamento. A medida da produção de leite foi realizada pelo método direto, com ordenha manual, seguindo a metodologia descrita por Ribeiro et al. (1991). As vacas foram separadas dos seus bezerros pela manhã - um dia antes da coleta - e mantidas em piquete anexo; às $18 \mathrm{~h}$, eram colocadas novamente com os bezerros, permanecendo com eles durante 30 minutos, tempo suficiente para esgotamento do úbere. Após esse tempo, os bezerros, separados de suas mães, permaneciam no curral até a manhã seguinte. A ordenha iniciava-se às seis horas, após aplicação de $3 \mathrm{~mL}$ de ocitocina via intramuscular, com o intuito de facilitar a descida do leite. A ordenha era realizada em dois quartos do úbere, um dianteiro e outro traseiro. A produção obtida, multiplicada por dois para obter a produção total do úbere, era ajustada para $24 \mathrm{~h}$, conforme a equação proposta por Restle et al. (2004b):

Produção de leite $(1 /$ dia $)=\frac{((\text { produção dos dois quartos do úbere }) * 2) * 60 \text { min } * 24 \mathrm{~h}}{\text { Tempo }(\min ) \text { entre a última mamada e a ordenha }}$

O delineamento experimental utilizado foi $\mathrm{o}$ inteiramente ao acaso, com número variável de repetições. Os dados coletados foram submetidos à análise de variância, incluindo no modelo os efeitos da suplementação. A ordem de parição e a idade da vaca foram utilizadas como covariáveis. As médias, quando diferentes significativamente, foram comparadas pelo teste t a 5\% de significância. Fez-se a análise da variável dependente do diagnóstico de gestação pelo método do $\chi^{2}$. As análises foram realizadas pelo programa estatístico SAS (2001).

\section{RESULTADOS E DISCUSSÃO}

A pastagem nativa apresentou massa de forragem média de 5006,56kg MS/ha, com teor de fibra em detergente neutro (FDN) de 78,7\% e proteína bruta (PB) de 4,4\%, composta na sua maior parte por capim-annoni -2 (Eragrostis plana Nees), gramínea cespitosa e perene de ciclo estival de origem africana, e espécies de estação quente como Paspalum notatum, Axonopus affinis e Desmodium incanum, as quais apresentam maior valor nutricional do que o capim-annoni. Tanure et al. (2011) observaram teor de PB de 8,6\%, 
utilizando pastagem nativa, com as mesmas espécies citadas acima e sem a presença do capim-annoni. Isto demonstra a possibilidade de os animais terem ingerido maior quantidade de nutrientes em razão, principalmente, de seleção por essas espécies no momento do pastejo.

O manejo alimentar não influenciou o peso e o ECC (P>0,05) (Tab. 1), o que mostra que a forragem consumida durante o período de gestação não foi limitante para o desempenho animal, nem a quantidade de sais de cálcio de ácidos graxos oferecida durante o pré-parto foi suficiente para incrementar o desempenho. Alguns estudos indicam que a nutrição pré-parto, que reflete o ECC ao parto, é um fator muito importante na determinação do tempo de retorno ao estro. Santos et al. (2009), ao avaliarem a influência do ECC sobre a eficiência reprodutiva de vacas de cria nos períodos pré e pós-parto, verificaram que, para obterem taxas de parição acima de $80 \%$, as vacas deviam apresentar ECC de 5,5 no pré-parto e de 6,0 no pós-parto, em uma escala de 1 a 9.

Tabela 1. Médias para o desempenho produtivo de vacas mantidas em pastagem natural ou suplementadas com gordura protegida no período pré e/ou pós-parto

\begin{tabular}{lcccc}
\hline \multirow{2}{*}{ Variável } & \multicolumn{4}{c}{ Manejo alimentar } \\
\cline { 2 - 5 } & PRE & POS & PREPOS & PN \\
\hline Peso ao parto, kg & 384,3 & 391,4 & 380,6 & 405,0 \\
Escore corporal ao parto, pts. & 2,45 & 2,52 & 2,49 & 2,52 \\
Peso à desmama, kg & 390,6 & 392,8 & 382,8 & 412,3 \\
Escore corporal à desmama, pts. & 2,52 & 2,60 & 2,58 & 2,61 \\
GMD parto-desmama, kg & 0,102 & 0,022 & 0,034 & 0,115 \\
Peso ao final da monta, kg & 409,7 & 417,1 & 406,8 & 429,5 \\
Escore corporal ao final da monta, pts. & $2,78 \mathrm{~b}$ & $2,96 \mathrm{a}$ & $2,88 \mathrm{ab}$ & $2,88 \mathrm{a}$ \\
GMD desmame - final de monta, $\mathrm{kg}$ & 0,307 & 0,392 & 0,388 & 0,277 \\
\hline
\end{tabular}

PRE= suplementação com gordura protegida (GP) 45 dias antes do parto; PREPOS: suplementação com GP 45 dias antes do parto e 63 dias pós-parto; POS: suplementação GP 63 dias pós-parto; PN: sem suplementação. GMD: ganho médio diário de peso. Médias seguidas por letras diferentes diferem entre si $(\mathrm{P}<0,05)$ pelo teste $\mathrm{t}$.

Restrição alimentar pré e pós-parto conduz a baixo peso ao parto e prolongado intervalo de anestro pós-parto, o que resulta em baixas taxas de prenhez. No presente estudo, observa-se que as vacas estavam em condições alimentares satisfatórias e que a quantidade e a qualidade das forrageiras foram suficientes para manter as vacas com boa condição corporal.

Da mesma forma, o peso e o ECC à desmama foram semelhantes $(\mathrm{P}>0,05)$ entre as vacas suplementadas ou não, com acréscimo de apenas 1,$6 ; 0,36 ; 0,6$ e $1,8 \%$ em relação ao peso ao parto, para os manejos alimentares PRE, POS, PREPOS e PN, respectivamente. As vacas foram desmamadas aos 63 dias de idade após o parto, e não responderam ao consumo de energia extra, possivelmente por ser pequeno o incremento energético via suplementação, uma vez que a adição da gordura protegida forneceu para as vacas do PRE $0,43 \mathrm{Mcal} / \mathrm{dia}$ e para as vacas do POS, 0,71Mcal/dia, enquanto a pastagem nativa apresentou 1,69Mcal EM/kg de MS.
O GMD do parto à desmama das vacas suplementadas ou não com lipídios protegidos não foi diferente $(\mathrm{P}>0,05)$. A quantidade e a qualidade da forragem disponível podem ter mascarado qualquer efeito possível da suplementação, uma vez que o GMD foi apenas ligeiramente mais alto $(\mathrm{P}>0,05)$ para as vacas não suplementadas no pós-parto. Bellows et al. (2001), em dois experimentos, suplementaram novilhas primíparas com sementes de cártamo, soja e girassol $-4,7 ; 3,8$; e $5,1 \%$ de gordura na dieta, respectivamente -, durante 65 dias antes do parto. Esses autores verificaram, no primeiro experimento, aumento na taxa de prenhez, mas, no segundo, não houve melhora na taxa de prenhez; assim, concluíram que a maior diferença entre os dois estudos foi a disponibilidade de forragem: $71 \%$ a mais de forragem disponível e maior qualidade nutricional no segundo estudo.

Na Tab. 1, o peso ao final da monta e o GDM do desmame ao final da monta (GMDdfm) foram semelhantes entre os manejos alimentares $(\mathrm{P}>0,05)$, e isto se deve, principalmente, à 
semelhança no GMD durante o período do parto ao desmame. O GMDdfm foi importante para o aumento de peso porque o GMD do parto ao desmame foi baixo, uma vez que as vacas estavam lactando, fase de maior demanda energética. De acordo com Restle et al. (2001), o peso final de monta é uma característica importante, pois maiores pesos no outono indicam melhor condição para atravessar o período crítico do inverno e apresentar melhor condição corporal na primavera, durante a parição subsequente.

Cantrell et al. (1981) verificaram que as perdas de peso após a parição, acima de $10 \%$, diminuem a taxa de concepção, portanto o ganho de peso das vacas após o parto foi determinante para o desempenho reprodutivo. Vacas que ganharam 101 a $500 \mathrm{~g}$ de peso vivo por dia, como encontrado no presente estudo, têm $80 \%$ de probabilidade de emprenharem na estação reprodutiva (Santos et al., 2009).

O ECC no final do período de acasalamento (Tab. 1) foi menor para as vacas do PRE $(\mathrm{P}<0,05)$. Em comparação com outros estudos realizados na mesmo local do presente experimento, observa-se que o ECC das vacas foi mais baixo (Cerdótes et al., 2004; Restle et al.,
2009), porém essa baixa condição não afetou a taxa de prenhez. O escore de condição corporal é uma medida visual, prática e de baixo custo, que é utilizada para avaliar o estado nutricional e o desempenho reprodutivo de matrizes, servindo para analisar práticas de manejo adotadas e também como subsídio para melhorar a eficiência reprodutiva e a nutricional dos rebanhos. Maciel et al. (2001) correlacionaram o ECC com as taxas de prenhez e não observaram associação entre essas características.

Vacas suplementadas com gordura protegida no PREPOS apresentaram maior produção de leite do que as suplementadas no PRE $(\mathrm{P}<0,05$; Tab. 2). Isto se deve ao melhor aproveitamento dos ácidos graxos de cadeia longa para a síntese do leite na glândula mamária (Nörnberg et al., 2006). As vacas não suplementadas na $P N$ produziram, em média, 5,88L/dia. Tais produções foram semelhantes à das vacas do PREPOS, mas estas últimas produziram 14,2\% a mais de leite. Van Knegsel et al. (2005) afirmam que há controvérsias quanto aos benefícios da inclusão de gordura protegida na dieta, pelo fato de os lipídios não serem os principais precursores da glicose, que é utilizada para a síntese de lactose na glândula mamária.

Tabela 2. Médias da produção e da composição química do leite de vacas mantidas em pastagem natural ou suplementadas com gordura protegida nos períodos pré e/ou pós-parto

\begin{tabular}{|c|c|c|c|c|}
\hline \multirow{2}{*}{ Variável } & \multicolumn{4}{|c|}{ Manejo alimentar } \\
\hline & PRE & POS & PREPOS & PN \\
\hline Produção de leite, litros & $5,62 b$ & $5,85 \mathrm{ab}$ & $6,85 a$ & $5,88 \mathrm{ab}$ \\
\hline Gordura, \% & 3,44 & 3,15 & 3,36 & 3,20 \\
\hline Proteína, \% & 2,74 & 2,70 & 2,85 & 2,69 \\
\hline Lactose, \% & $4,64 \mathrm{ab}$ & $4,69 a$ & $4,43 b$ & $4,69 \mathrm{ab}$ \\
\hline Sólidos totais, $\%$ & 11,77 & 11,49 & 11,56 & 11,53 \\
\hline Energia do leite, Mcal/dia ${ }^{1}$ & $4,08 \mathrm{ab}$ & $3,84 \mathrm{ab}$ & $4,68 \mathrm{a}$ & $4,03 \mathrm{~b}$ \\
\hline
\end{tabular}

PRE= suplementação com gordura protegida (GP) 45 dias antes do parto; PREPOS: suplementação com GP 45 dias antes do parto e 63 dias pós-parto; POS: suplementação GP 63 dias pós-parto; PN: sem suplementação. Médias seguidas por letras diferentes diferem entre si $(\mathrm{P}<0,05)$ pelo teste $t$.

${ }^{1}$ Energia do leite (Mcal/kg produzido) calculada segundo o NRC (Nutrient..., 1996): EL= $(0,097 * \%$ gordura do leite) $+0,361$.

${ }^{\#}$ Análise de contraste: vacas não suplementadas vs. vacas suplementadas $(\mathrm{P}<0,05)$.

Entre os componentes químicos do leite, apenas o teor de lactose foi diferente (Tab.2; $\mathrm{P}<0,05)$. As vacas alimentadas no PREPOS apresentaram menor teor de lactose no leite $(4,4 \%)$ do que as do POS $(4,7 \%)$, mas esse valor não diferiu dos obtidos nos tratamentos PN e PRE. A lactose e o potássio no leite mantêm o equilíbrio osmótico entre o leite e o sangue mediante a retirada de água dos fluidos extra e intracelulares. Dessa forma, quanto mais lactose é secretada, tanto mais água é necessária para formar o leite $(87,5 \%)$, fazendo com que a lactose seja o componente do leite menos afetado pela alimentação (Mühlbach, 2000). Duske et al. 
(2009), ao suplementarem vacas durante 12 semanas pré-parto com gordura, verificaram redução na produção de leite e menor teor de lactose.

$\mathrm{Na}$ análise de contrastes, as vacas suplementadas apresentaram maior teor de sólidos totais do leite do que as não suplementadas. Os sólidos totais equivalem ao somatório de todos os componentes, com exceção da água; dessa forma, este resultado era esperado, uma vez que os maiores valores dos componentes químicos foram encontrados nas vacas suplementadas.

A suplementação com gordura protegida não influenciou $\mathrm{o}$ peso ao nascer e o peso ao desmame dos bezerros ( $\mathrm{P}>0,05$; Tab. 3), bem como o seu GMD do nascimento à desmama. Era esperada diferença no ganho médio diário dos bezerros e, consequentemente, no peso ao desmame, uma vez que houve diferença entre os tratamentos para a produção de leite das mães, e na literatura vários estudos indicam que a produção de leite é um dos principais fatores que influenciam o peso à desmama dos bezerros (Jenkis et al., 1991; Fiems et al., 2008). Vaz et al. (2011), ao avaliarem duas idades de desmame, aos 80 e 152 dias, de bezerros Braford mantidos em pastagem nativa, observaram, ao desmame precoce, média de peso de 89,1 e $84,7 \mathrm{~kg}$, respectivamente. Brauner et al. (2011), ao suplementarem vacas de corte mantidas em pastagem natural durante 35 dias pós-parto, também não encontraram diferença no desempenho dos bezerros filhos de vacas não suplementadas.

O peso aos 205 dias e o GMD do nascimento até aos 205 dias foram similares entre os manejos alimentares testados ( $\mathrm{P}>0,05$; Tab. 2). Ressaltase que o elevado GMD do nascimento ao desmame sugere que a produção de leite das vacas foi primordial para este desempenho elevado, uma vez que os bezerros estavam em pastagem nativa que, provavelmente, não fazia parte de sua dieta.

Tabela 3. Médias para o desempenho de bezerros e litros de leite produzido pela vaca por quilo de ganho de peso do bezerro (PL/GPB) de vacas mantidas em pastagem natural ou suplementadas com gordura protegida nos períodos pré e/ou pós-parto

\begin{tabular}{lcccc}
\hline \multirow{2}{*}{ Variável } & \multicolumn{4}{c}{ Manejo alimentar } \\
\cline { 2 - 5 } & PRE & POS & PREPOS & PN \\
\hline Peso ao nascer, $\mathrm{kg}$ & 34,7 & 34,1 & 35,2 & 34,0 \\
Peso ao desmame, kg & 82,5 & 84,3 & 85,1 & 83,9 \\
GMD do nascimento ao desmame, $\mathrm{kg}$ & 0,758 & 0,796 & 0,793 & 0,788 \\
Peso aos 205 dias, kg & 165,4 & 160,6 & 162,1 & 166,0 \\
GMD do nascimento aos 205 dias, kg & 0,637 & 0,617 & 0,620 & 0,644 \\
PL/GPB, 1/kg & 7,40 & 7,66 & 7,40 & 7,81 \\
\hline
\end{tabular}

$\mathrm{PRE}=$ suplementação com gordura protegida (GP) 45 dias antes do parto; PREPOS: suplementação com GP 45 dias antes do parto e 63 dias pós-parto; POS: suplementação GP 63 dias pós-parto; PN: sem suplementação; GMD= ganho de peso médio diário.

Houve similaridade $(\mathrm{P}>0,05)$ na eficiência de transformar litros de leite em ganho de peso (Tab. 3). No presente estudo, $58 \%$ da variação no peso à desmama dos bezerros são atribuídos ao consumo de leite, corroborando os dados de outras pesquisas, que encontraram correlações semelhantes (Restle et al., 2004b; Fiems et al., 2008). Restle et al. (2004b) observaram que bezerros mantidos em pastagem cultivada foram mais eficientes em relação aos bezerros mantidos em pastagem nativa, $11,0 \mathrm{~L} / \mathrm{kg}$, e atribuíram estes resultados às diferenças ocorridas na produção de leite e, principalmente, ao ganho de peso médio diário dos bezerros.
A suplementação não afetou a taxa de prenhez ( $\mathrm{P}>0,05$; Tab.4), provavelmente pela semelhança entre os ganhos de peso das vacas ao longo do experimento. Sklan et al. (1991) também não encontraram melhora na taxa de prenhez de vacas de corte alimentadas com gordura. A taxa de prenhez do PN foi mais alta que os $66,7 \%$ relatados por Lobato et al. (1998) em novilhas mantidas em campo natural, o que pode ser atribuído à disponibilidade e à seleção de pasto de melhor qualidade. 
Na duração média do intervalo entre partos não foram observadas diferenças entre PREPOS e PN, mas PREPOS apresentou intervalo menor que PRE e POS $(\mathrm{P}<0,05)$. O tratamento $\mathrm{PN}$ não diferiu do PRE e do POS (Tab. 4). O tratamento PREPOS proporcionou melhor nível nutricional no pré e no pós-parto, determinando intervalo entre partos próximo do ideal para a obtenção de uma cria a cada ano, segundo recomendação de Sacco et al. (1990).

A utilização de gordura favoreceu o desempenho reprodutivo, independentemente de sua contribuição energética, pois os ácidos graxos podem modificar algumas vias específicas e influenciar o metabolismo de alguns hormônios - esteroides e eicosanoides - que modulam os processos metabólicos nos ovários e no útero, além de exercer efeitos diretos na transcrição de genes que codificam proteínas essenciais à reprodução, conforme sugeriu Funston (2004).

As vacas do PREPOS apresentaram, de modo geral, as melhores médias para eficiência produtiva. As vacas do PREPOS desmamaram $22,6 \mathrm{~kg}$ de bezerro para cada $100 \mathrm{~kg}$ de peso vivo da vaca ao parto, não diferiram das vacas mantidas no PRE e no POS. Em comparação as vacas não suplementadas, as vacas do PREPOS desmamaram $8,4 \%$ mais quilos de bezerro para cada $100 \mathrm{~kg}$ de vaca ao parto. Valores semelhantes também ocorreram quando a eficiência foi medida pelo peso das vacas ao desmame. Isto ocorreu porque as vacas dos tratamentos PRE, POS e PREPOS foram mais leves que as do PN e por não ter havido diferença nos pesos dos bezerros.

Tabela 4. Médias para eficiência produtiva e reprodutiva de vacas mantidas em pastagem natural ou suplementadas com sais de cálcio de ácidos graxos nos períodos pré e/ou pós-parto

\begin{tabular}{|c|c|c|c|c|}
\hline \multirow{2}{*}{ Variável } & \multicolumn{4}{|c|}{ Manejo alimentar } \\
\hline & PRE & POS & PREPOS & PN \\
\hline Taxa de prenhez, $\%$ & 78,9 & 91,3 & 82,6 & 85,7 \\
\hline EFRTM $^{1}$ & $74,4 \mathrm{c}$ & $87,3 \mathrm{a}$ & $81,7 \mathrm{ab}$ & $78,5 \mathrm{bc}$ \\
\hline Intervalo entre partos, dias & $386 a$ & $387 \mathrm{a}$ & $376 b$ & $383 a b$ \\
\hline EFPARTO $^{1}$ & $21,6 \mathrm{ab}$ & $21,6 \mathrm{ab}$ & $22,6 a$ & $20,7 b$ \\
\hline EFDESMAME $^{1}$ & $21,3 \mathrm{ab}$ & $21,6 \mathrm{ab}$ & $22,5 \mathrm{a}$ & $20,4 b$ \\
\hline \multicolumn{5}{|c|}{$\begin{array}{l}{ }^{1} \text { EFPARTO }=\text { eficiência produtiva ao parto }=(\mathrm{P} 63 / \mathrm{PVP}) * 100 ; \text { EFDESMAME }=\text { eficiência produtiva ao } \\
\text { desmame }=(\mathrm{P} 63 / \mathrm{PVD}) * 100 ; \text { EFRTM= eficiência reprodutiva em relação ao tamanho metabólico=(P63/(PVD } \\
\text { de prenhez. } \\
\text { PRE= suplementação com gordura protegida }(\mathrm{GP}) 45 \text { dias antes do parto; PREPOS: suplementação com GP } 45 \text { dias } \\
\text { antes do parto e } 63 \text { dias pós-parto; POS: suplementação GP } 63 \text { dias pós-parto; PN: sem suplementação. Médias } \\
\text { seguidas por letras diferentes diferem entre si }(\mathrm{P}<0,05) \text { pelo teste } t .\end{array}$} \\
\hline
\end{tabular}

Estes resultados corroboram os resultados de Ribeiro et al. (2001) e Euclides Filho et al. (1995), que atribuíram a melhor produtividade às vacas mais leves. Restle et al. (2007) verificaram que o melhor nível nutricional é o fator que proporciona melhores resultados de eficiência biológica. As vacas dos tratamentos POS e PREPOS apresentaram as melhores $(\mathrm{P}<0,05)$ médias de eficiência reprodutiva ajustada para o tamanho metabólico das vacas, enquanto as dos tratamentos PRE e PN apresentaram as menores médias e foram semelhantes entre si. Atribui-se essa maior eficiência reprodutiva às maiores taxas de prenhez nestes manejos alimentares.

\section{CONCLUSÕES}

A suplementação com gordura protegida durante os períodos pré e/ou pós-parto não afetou o desempenho de vacas e de bezerros. A composição e a produção média de leite de vacas não são afetadas pelo fornecimento de gordura protegida. Gordura protegida fornecida durante o período pós-parto aumenta a eficiência produtiva das vacas de corte. 


\section{REFERÊNCIAS}

BELLOWS, R.A.; GRINGS, E.E; SIMMS, D.D. et al. Effects of feeding supplemental fat during gestation to first-calf beef heifers. Profess. Anim. Sci., v.17, p.8189, 2001

BRAUNER, C.C.; PIMENTEL, M.A.; MENEZES, L.M. et al. Effect of short period feed supplementation during early lactation on performance of cows and calves raised in extensive system. Rev. Bras. Zootec., v.40, p.1381-1387, 2011.

BUTLER, W.R. Nutritional interactions with reproductive performance in dairy cattle. Anim. Reprod. Sci., v.60, p.449-457, 2000.

CANTRELL, J.A.; KROPP, J.R.; ARMBRUSTER, S.L. et al. he influence of postpartum nutrition and weaning age of calves on cow body condition, estrus, conception rate and calf performance of fall-calving beef cows. Oklahoma Agric. Experim. Station (Animal Science Research Report). MP 112, p.53-58, 1981.

CÉRDOTES, L.; RESTLE, J.; BRONDANI, I.L. et al. Desempenho produtivo de vacas de quatro grupos genéticos submetidas a diferentes manejos alimentares desmamadas aos 42 ou 63 dias pós-parto. Rev. Bras. Zootec., v.33, p.585-596, 2004

COLAZO, M.G.; HAYIRLI, A.; DOEPEL, L. et al. Reproductive performance of dairy cows is influenced by prepartum feed restriction and dietary fatty acid source. J. Anim. Sci., v.92, p.2562-2571, 2009.

DUSKE, K.; HAMMON, H.M.; LANGHOF, A-K. et al. Metabolism and lactation performance in dairy cows fed a diets containing rumen-protected fat during the last twelve weeks of gestation. J. Dairy Sci., v.92, p.1670-1684, 2009.

EUCLIDES FILHO, K.; FIGUEIREDO, G.R.; EUCLIDES, V.P.B. Eficiência de produção de vacas de corte com diferentes potenciais para produção de leite. Pesq. Agropec. Bras., v.30, p.1003-1007, 1995.

FIEMS, L.O.; VAN CAELENBERGH, W.; CAMPENEERE, S.D. et al. Effect of dam factors on milk intake and performance of Belgian Blue suckling calves. Anim., v.2, p.135-140, 2008.

FUNSTON, R.N. Fat supplementation and reproduction in beef females. J. Anim. Sci., v.82, p.154-161, 2004

JENKIS, T.G.; CUNDIFF, L.V.; FERREL, C.L. Diferences among breed crosses of cattle in the conversion of food energy to calf weight during the preweaning interval. J. Anim. Sci., v.69, p.2762-2769, 1991.
JENKIS, T.G; FERREL, C.L. Lactation characteristics of nine breeds of cattle fed various quantities of dietary energy. J. Anim. Sci., v.70, n.6, p.1652-1660, 1992.

LOBATO, J.F.P.; ZANOTTA JÚNIOR, R.L.D.; PEREIRA NETO, O.A. Efeitos das dietas pré e pósparto na eficiência reprodutiva de vacas primíparas de corte. Rev. Bras. Zootec., v.27, p.857-862, 1998.

LOPES, C.N.; SCARPA, A.B.; CAPPELLOZZA, B.I. et al. Effects of rumen-protected polyunsaturated fatty acid supplementation on reproductive performance of Bos indicus beef cows. J. Anim. Sci., v.87, p.39353943, 2009.

LOWMAN, B.G.; SCOTT, N.; SOMERVILLE, S. Condition scoring beef cattle. Edinburg: East of Scotland College of Agriculture, 8p. (Bulletin, 6), 1973.

MACIEL, M.N.; NEVES, J.P.; GONÇALVES, P.B.D. et al. Efeito da somatotropina bovina (bST-r), do implante de progestágeno e do desmame por 72 horas na indução do estro e na taxa de prenhez em vacas. Arq. Bras. Med. Vet. Zootec., v.53, p.666-670, 2001

MÜHLBACH, P.R.F.; OSPINA, H.; PRATES, E.R. et al. Aspectos nutricionais que interferem na qualidade do leite. In: ENCONTRO ANUAL DA UFRGS SOBRE NUTRIÇÃO DE RUMINANTES, 2000, Porto Alegre. Anais...Novos desafios para a produção leiteira do Rio Grande do Sul. Porto Alegre: 2000. p.73-102.

NÖRNBERG, J.L.; LÓPEZ, J.; STUMPF JÚNIOR, W. et al. Desempenho de vacas Jersey suplementadas com diferentes fontes lipídicas na fase inicial de lactação. Rev. Bras. Zootec., v.35, p.1431-1438, 2006.

NUTRIENT requirements of beef cattle. 7.ed. Washington: National Academy of Sciences, 1996. 242p.

PALMQUIST, D.L.; JENKIS, T.C. Fat in lactation rations: review. J. Dairy Sci., v.63, p.1-14, 1980.

RESTLE, J.; VAZ, R.Z.; ALVES FILHO, S.C. Desempenho de vacas Charolês e Nelore desterneiradas aos três ou sete meses. Rev. Bras. Zootec., v.30, p.499-507, 2001.

RESTLE, J.; PACHECO, P.S.; PÁDUA, J.T. et al. Eficiência biológica de vacas de dois grupos genéticos amamentando bezerros puros ou F1, mantidas em diferentes condições de alimentação. Rev. Bras. Zootec., v.33, p.1822-1832, 2004a.

RESTLE, J.; PACHECO, P.S.; PÁDUA, J.T. et al. Efeito da pastagem, da produção e da composição do leite no desempenho de bezerros de diferentes grupos genéticos. Rev. Bras. Zootec., v.33, p.691-703, 2004b. 
RESTLE, J.; PACHECO, P.S.P.; FREITAS, A.K. et $a l$. Influência das taxas de ganho de peso pré-desmame das vacas e do tipo de pastagem no período pós-parto sobre a eficiência biológica de vacas e de bezerros de corte. Rev. Bras. Zootec., v.36, p.874 - 880, 2007.

RESTLE, J.; VAZ, R.Z.; PASCOAL, L.L. et al. Desenvolvimento e desempenho reprodutivo de novilhas de corte submetidas a diferentes idades de desmame. Cienc. Anim. Bras., v.10, p.808-817, 2009.

RIBEIRO, E.L.A.; RESTLE, J. Desempenho de bezerros Charolês e Abeerden Angus puros e seus mestiços com Nelore. Pesq. Agropec. Bras., v.26, p.1145-1151, 1991.

RIBEIRO, E.L.A.; RESTLE, J.; ROCHA, M.A. et al. Eficiência produtiva em vacas primíparas das raças Aberdeen Angus e Charolês. Rev. Bras. Zootec., v.30, p.125-132, 2001.

RICHARDS, M.W.; SPITZER, J.C.; WARNER, M.B. Effect of varying levels of postpartum nutrition and body condition at calving on subsequent reproductive performance in beef cattle. J. Anim. Sci., v.62, p.300, 1986.

SACCO, R.E.; BAKER, J.F.; CARTWRIGHT, T.C. et al. Measurements at calving for straightbred and crossbred cows of diverse types. J. Anim. Sci., v.68, p.3103-3108, 1990.
SANTOS, S.A.; ABREU, U.G.P.; SOUZA, G.S. et al. Condição corporal, variação de peso e desempenho reprodutivo de vacas de cria em pastagem nativa no Pantanal. Rev. Bras. Zootec., v.38, p.354-360, 2009.

SAS. Statistical Analysis Systems User's Guide. Version 2001, SAS Institute, Cary, NC.

SKLAN, D.; MOALLEM, U.; FOLMAN, Y. Effect of feeding calcium soaps of fatty acids on production and reproductive responses in high producing lactating cows. J. Dairy Sci., v.74, p. 510-517, 1991.

TANURE, S.; PÖTER, B.A.A; LOBATO, J.F.P. Natural and improved natural pastures on the reproductive performance of first-calf beef cows. Rev. Bras. Zootec., v.40, p.690-699, 2011.

VAN KNEGSEL, A.T.; VAN DEN BRAND, H.; DIJKSTRA, S. et al. Effect of dietary energy source on energy balance, production, metabolic disorders and reproduction in lactating dairy cows. Reprod. Nutr. Dev., v.45, p.665-688, 2005.

VAZ, R.Z.; LOBATO, J.F.P.; PASCOAL, L.L. Desenvolvimento de bezerros de corte desmamados aos 80 e 152 dias até os 15-16 meses de idade. Rev. Bras. Zootec., v.40, p.221-229, 2011.

WILM, H.G.; COSTELlO, D.F.; KLIPPLE, G.E. Estimating forage yield by the double-sampling methods. J. Am. Soc. Agron., v.36, p.194-203, 1944. 